(2) Open Access Full Text Article

\title{
Pharmacokinetic interactions and tolerability of berberine chloride with simvastatin and fenofibrate: an open-label, randomized, parallel study in healthy Chinese subjects
}

\author{
This article was published in the following Dove Medical Press journal: \\ Drug Design, Development and Therapy
}

\author{
Guofei Li \\ Mingming Zhao \\ Feng Qiu \\ Yaxin Sun
}

Limei Zhao

Department of Pharmacy, Shengjing Hospital of China Medical University, Shenyang II0004, China
Correspondence: Limei Zhao Shengjing Hospital of China Medical University, 36 Sanhao Street, Shenyang, Liaoning 110004 , China

Tel/fax +862423925I08

Email ligfpharm@sina.com
Purpose: Fenofibrate (Fbt) is a prodrug that has been used to reduce low-density-lipoprotein cholesterol, triglycerides, and increase high-density-lipoprotein cholesterol. Simvastatin (Svt) is a classic lipid-lowering drug that is widely used in the treatment of hypercholesterolemia and hypertriglyceridemia, while berberine chloride (Bbr) is a novel hypolipidemic agent and its blood-lipid-reducing mechanism is distinct from traditional drugs. Currently, drug combination is the trend in treating hyperlipidemia to improve clinical efficacy. The purpose of this study was to evaluate drug interaction from the perspective of pharmacokinetics between $\mathrm{Bbr}$ and $\mathrm{Fbt} / \mathrm{Svt}$ and the tolerability of combined administration in healthy Chinese subjects.

Methods: Healthy subjects $(n=60)$ were randomly allocated to five treatment groups: Bbr alone, Fbt alone, Svt alone, Bbr plus Fbt, and Bbr plus Svt. The experiment was divided into two parts: single-dose administration and multiple-dose administration. Bbr, Fbt, and Svt were taken once every 8 hours, 24 hours, and 24 hours, respectively, over 7 days in the multidose group. Plasma samples were collected and liquid chromatography-mass spectrometry/mass spectrometry was used to detect drug concentrations.

Results: No serious adverse reactions or intolerance were observed throughout the trial. More importantly, the combined-administration groups did not show an increase in incidence of side effects. Coadministration of Fbt and Svt with Bbr had no significant effect on the pharmacokinetic parameters of Bbr, except time to maximum concentration, apparent volume of distribution, and apparent clearance. Concurrent coadministration of Bbr had no obvious impact on the pharmacokinetic behavior of Fbt or Svt. Additionally, there was no significant correlation between sex and pharmacokinetic results.

Conclusion: All treatments were well tolerated. No clinically obvious pharmacokinetic interactions between $\mathrm{Bbr}$ and $\mathrm{Fbt} / \mathrm{Svt}$ were observed with combined administration. The results demonstrated that Bbr can be coadministered safely with Fbt and Svt without dose adjustment.

Keywords: Bbr, Fbt, Svt, drug-drug interaction, pharmacokinetics

\section{Introduction}

Hyperlipidemia has now become an important risk factor threatening human health with the improvement in living standards, which is closely related to lots of diseases, such as myocardial infarction, cerebral infraction, stroke, and hemiplegia. ${ }^{1-3}$ Therefore, timely detection and drug intervention is of great significance for hyperlipidemia.

Currently, there are many types of drugs for regulating blood lipids, but all have obvious therapeutic limitations and side effects. Simvastatin (Svt) is a 
3-hydroxy-3-methylglutaryl coenzyme A reductase inhibitor that is more effective at reducing total cholesterol and lowdensity lipoprotein when used at a low dose. ${ }^{4}$ As such, it is the most potent drug for treating hyperlipidemia. However, Svt monotherapy does not always achieve the desired effects, due to the complexity of patients with hyperlipidemia (including disease factors, age, nutrition, and liver function), particularly those with severe hyperlipidemia. ${ }^{5}$ Fenofibrate (Fbt) is another type of drug commonly used to regulate blood lipids, and can reduce the level of triglycerides and low-densitylipoprotein cholesterol by increasing lipoprotein lipase activity to accelerate the breakdown of very low-density lipoprotein. ${ }^{6}$ Similarly, Fbt monotherapy cannot achieve a satisfactory therapeutic effect, due to its poor cholesterollowering ability. ${ }^{7}$ It can be seen that there is currently no single drug that can fully regulate blood lipids. Therefore, to meet this treatment need, it may be necessary to combine other drugs with different mechanisms of action to improve efficacy and avoid the risk of treatment in the clinic.

Berberine (Bbr) hydrochloride is an isoquinoline alkaloid of the protoberberine type, and it has been found to have a clear hypolipidemic effect in recent years. ${ }^{8}$ The mechanism of Bbr in lowering blood lipids is significantly different from traditional lipid-lowering drugs. Firstly, Bbr can activate the ERK pathway, increasing the expression of low-density-lipoprotein receptors, prolonging the half-life of low-density-lipoprotein receptor mRNA and stabilizing the mRNA thereof. ${ }^{9}$ Secondly, Bbr can reduce liver lipid peroxide, triglycerides, and total-cholesterol levels, and its effects are equivalent to or superior to stains. This is mainly because Bbr can inhibit the phosphorylation of acetyl-CoA carboxylase through the AMPK pathway, thereby inhibiting the synthesis of lipids in hepatocytes. Thirdly, Bbr can prevent the absorption of lipids and relipidation of free fatty acids and promote the catabolism of lipids. ${ }^{10}$ More importantly, Bbr shows lower side effects and good tolerance when administrated orally compared with other drugs. ${ }^{11}$ Therefore, Bbr is expected to be part of a new generation of highly effective drugs for regulating blood lipids. Meanwhile, the combination of Bbr and other lipid-lowering drugs not only regulates blood lipids through multiple targets but also reduces the occurrence of side effects. For example, a study has shown that the combination of Bbr and Svt is significantly better than Svt alone in terms of lowering serum low-densitylipoprotein cholesterol. Furthermore, the Svt dose can be reduced by at least $40 \%$ when Bbr and Svt are combined. ${ }^{12}$ Although there have been no reports on the combination of $\mathrm{Bbr}$ and $\mathrm{Fbt}$, it can be concluded that their combination may have broader application prospects in view of the serious side effects of Fbt. Therefore, combined drug therapy for hyperlipidemia has important clinical significance and will become the mainstream strategy in the future.

It has become very important to obtain in vivo information on drug interactions among numerous lipid-lowering drugs from a safety aspect, as multiple-drug therapy is commonly used in clinical practice. ${ }^{13-15}$ For example, the pharmacokinetic behavior of cyclosporine is significantly affected in a dose-dependent manner after the combination of cyclosporine and Bbr. A study found that area under the curve (AUC) and maximum concentration $\left(C_{\max }\right)$ of cyclosporine increased by $96 \%$ and $60 \%$, respectively, when the Bbr dose was $100 \mathrm{mg} / \mathrm{kg}$, which greatly increased the risk of cyclosporine poisoning. ${ }^{16}$ This is mainly because Bbr inhibits the activity of the CYP3A4-metabolizing enzyme, resulting in reduced metabolism of its substrate - cyclosporine. Similarly, the absorption of Bbr is significantly decreased when Bbr and baicalin are combined. But the bioavailability of baicalin obviously improved with the presence of Bbr. ${ }^{17}$ It is well known that CYP3A4 and OATP1BA are metabolic enzymes and transporters, respectively, of Bbr, while baicalin has a significant induction effect on CYP3A4 and OATP1BA, which ultimately leads to the occurrence of drug interactions. It can be seen that the interaction among drugs must cause us to pay enough attention, especially drug interactions mediated by metabolic enzymes and transporters.

Metabolic enzymes and transporter-mediated drug interactions are the most common and most important forms of interaction. ${ }^{18-20} \mathrm{Bbr}$ is mainly metabolized by CYP1A2, CYP2D6, and CYP3A4 and is also a substrate of Pgp and OCT2. ${ }^{21}$ Svt is also a substrate of CYP3A4, but has an inhibitory effect on CYP $3 \mathrm{~A} 4,{ }^{22}$ while Fbt is metabolized primarily by CYP3A4 and glucuronosyltransferases. ${ }^{23}$ Meanwhile, Fbt is capable of inhibiting the activity of OCT2-mediated organic transporters. ${ }^{23}$ It can be concluded that drug interactions may occur among Bbr, Svt, and Fbt because of their obvious overlapping of metabolic enzymes and transporters. Therefore, the primary purpose of this paper was to evaluate the pharmacokinetic interactions and tolerability of Bbr and Svt or Fbt in combined administrations in healthy Chinese volunteers to improve the safety of clinical medications.

\section{Methods}

\section{Drug information}

All test drugs are commonly used in similar preparations. Among them, Svt tablets are developed and produced by Merck with a specification of $40 \mathrm{mg}$. Fbt capsules are 
produced by Recipharm Fontaine and its specification is $200 \mathrm{mg}$. Bbr tablets are produced by Northeast Pharmaceutical with a specification of $100 \mathrm{mg}$.

\section{Subjects}

All subjects provided written informed consent prior to screening. These studies were conducted and monitored in accordance with the ethical and scientific principles required by the Declaration of Helsinki and Chinese good clinical practice. All procedures were ethically and scientifically approved by the ethics committee of Shengjing Hospital of China Medical University (2015PS36, 2015-08-27). This clinical trial complied with the design and regulations of this program.

Healthy male and female volunteers aged $18-50$ years with a body-mass index of $18-26 \mathrm{~kg} / \mathrm{m}^{2}$ and total body weight $>45 \mathrm{~kg}$ (female) and $>50 \mathrm{~kg}$ (male) were eligible for this study. The ratio of men to women was 1:1. All subjects were considered to be in good health, based on hospital examinations, medical history inquiry, physical examination, blood pressure, breathing, body temperature, heart rate, 12-lead electrocardiography, clinical laboratory tests (hematology examination, blood biochemical examination, and urinalysis examination), serum virological examination (hepatitis B surface antigen, hepatitis $\mathrm{C}$ antibodies, and HIV antigen/antibodies), pregnancy tests, and urine drug screening (methamphetamine, cocaine, heroin, phenobarbital, caffeine, cannabinoids, and nicotine) within 2 weeks before the start of the clinical study. Subjects who had recently applied any experimental or similar drug, were allergic to experimental drugs, had a pregnancy planned, or showed a history of drug abuse were excluded. The investigators truthfully informed subjects of the risks and benefits of the trial. In addition, subjects had to have signed an informed-consent document before the start of the trial.

\section{Study design}

This was a phase I, single center, open-label, randomized, parallel, single/multiple-dose clinical trial (Figure 1). The trial consisted of five groups: Bbr $300 \mathrm{mg}$, Svt $40 \mathrm{mg}$, Fbt $200 \mathrm{mg}$, Bbr $300 \mathrm{mg}$ plus Svt $40 \mathrm{mg}$, and Bbr $300 \mathrm{mg}$ plus Fbt 200 mg. SAS 9.4 was used to randomize the subjects. During the screening phase, each subject would be identified using a screening number. After screening, randomization was performed prior to trial administration. Each eligible subject would receive a randomized number, which would be randomized according to the screening number from small to large. Randomized subjects who withdrew or were withdrawn from clinical trials for any reason would retain their randomized number, and the subject would not be allowed to reenter the trial. Before the start of the trial, if the subject dropped out for any reason or the number of subjects did not meet statistical requirements, subjects who had passed the pretest physical examination but were not enrolled would replace them, and these subjects would get a new randomization number (discarded volunteer randomization number plus 100). The replacement subject and the shed subject received the same treatment sequence and maintained the balance of the sequence. Eligible subjects admitted to the phase I trial ward on the day before the first

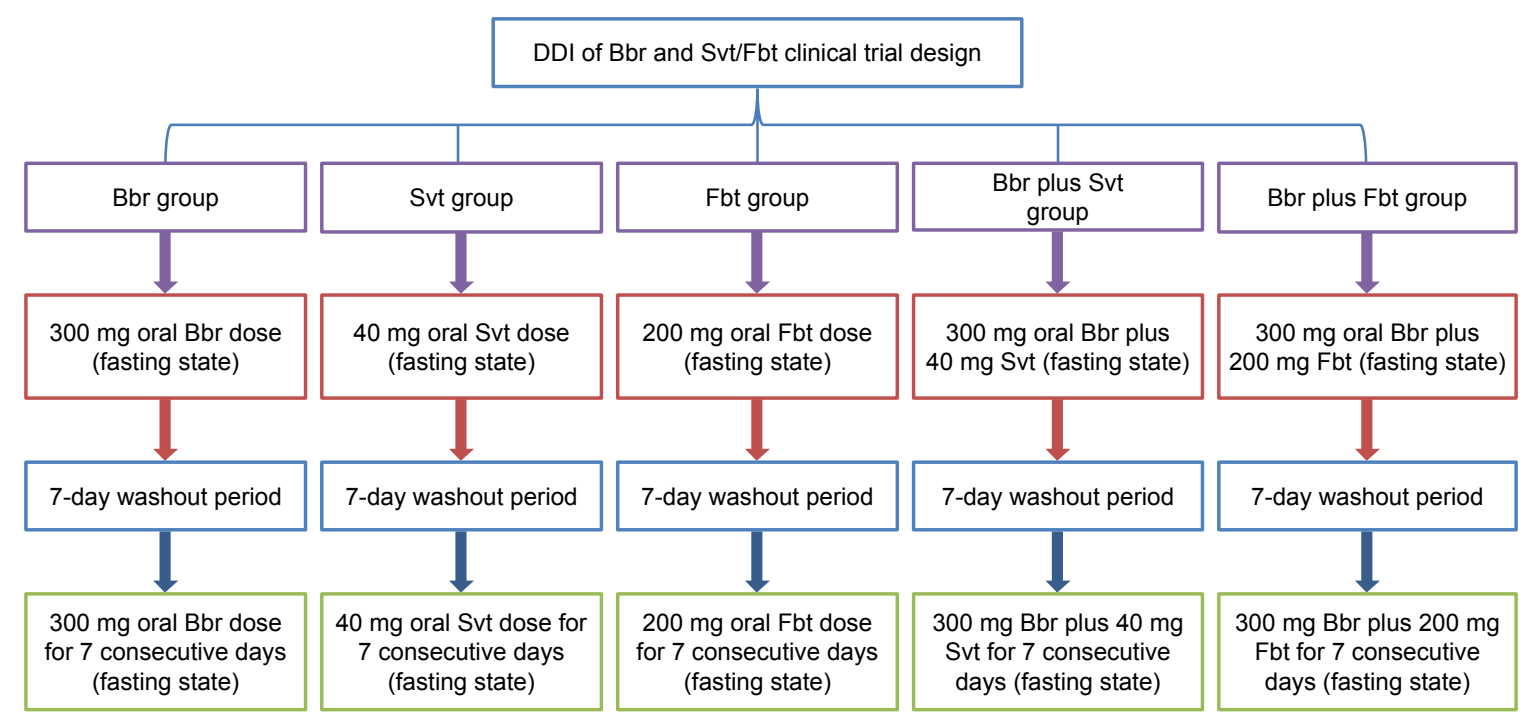

Figure I Study design.

Abbreviations: DDI, drug-drug interaction; Bbr, berberine chloride; Svt, simvastatin; Fbt, fenofibrate. 
dose were randomly assigned to one of the five groups and accepted the corresponding drug on the morning of day 1. All treatments were carried out under an overnight-fasted condition along with $200 \mathrm{~mL}$ water and were given in a single dose of $300 \mathrm{mg}$ Bbr, $40 \mathrm{mg}$ Svt, $200 \mathrm{mg}$ Fbt, $300 \mathrm{mg}$ Bbr plus 40 mg Svt, or 300 mg Bbr plus $200 \mathrm{mg}$ Fbt. Blood samples were collected in heparinized tubes prior to the drug-administration zero time and at $0.5,1,1.5,2,3,4,6,8$, $12,24,36,48,72,96$, and 120 hours. After a 7-day washout period, the subjects were given Bbr, Svt, Fbt, Bbr plus Svt, or Bbr plus Fbt for 7 days continuously. Among these, doses of Bbr, Svt, and Fbt were still $300 \mathrm{mg}, 40 \mathrm{mg}$, and $200 \mathrm{mg}$, and administration intervals were 8 hours, 24 hours, and 24 hours, respectively. Blood samples were collected in heparinized tubes at -3 days, -2 days, and -1 day at drug administration and at $0.5,1,2,3,4,6,8,12,24,36,48,60$, 72,96 , and 120 hours. Blood samples $(3 \mathrm{~mL})$ were centrifuged (4,000 rpm/10 minutes), and the plasma collected was stored at $-70^{\circ} \mathrm{C}$ until determination.

\section{Safety assessments}

Ensuring the safety of subjects is a top priority in clinical trials. Here, safety was monitored throughout the study from signing of the informed-consent document until study completion. Also, subject safety was confirmed by follow-up telephone calls after the end of the trial. Safety evaluation consisted of conventional physical examinations, assessment of adverse events (AEs), and serious AEs (SAEs), 12-lead electrocardiography, and clinical laboratory safety tests. AEs and SAEs were recorded in detail, including symptoms, severity, duration, relevance to the trial drugs, and outcome. In this study, AEs were divided into three levels: mild (subjects can tolerate, do not affect normal functions, no special treatments needed), moderate (unbearable, normal functions affected to some extent, necessary to stop taking medicine or receive special treatment), and severe (life-threatening, injury or disability, requiring immediate withdrawal or urgent treatment). Generally speaking, investigators should pay attention to the determination of AE severity when investigating AEs. AE-evaluation criteria were: 1) Is there a reasonable time relationship between medication and AEs?; 2) Does the reaction meet the known type of adverse reaction of the drug?; 3) After the drug is stopped or reduced, will the AEs disappear or be alleviated?; 4) Are the same AEs occurring again after using suspicious drugs again?; 5) Can AEs be explained by the effects of combined medication, progression of the patient's condition, and the effects of other treatments? Grading standards were: 1) certainly relevant, ie, the time sequence is reasonable and the reaction consistent with the adverse reactions of known drugs, the reaction disappears or is alleviated after stopping the drug or reappears after readministration, and it cannot be reasonably explained by the combined drug and the patient's disease; 2) most likely related, ie, the time sequence is reasonable and the reaction consistent with the adverse reactions of known drugs, the reaction disappears or is relieved after stopping the drug, and it cannot be reasonably explained by the drug combination or the patient's disease, or the time sequence is reasonable and the reaction is not an adverse reaction to known drugs, the reaction disappears or is relieved after stopping the drug and reappears after readministration, and it cannot be reasonably explained by the combined drug and the patient's disease (new AEs); 3) may be related, ie, the chronological order is reasonable and the response consistent with known adverse drug reactions and disease or other treatments can also cause such results, or the time sequence is reasonable and the reaction is not a known adverse drug reaction, and according to the actual clinical situation, the occurrence of adverse reactions is highly correlated with the drug (new AEs); and 4) may be unrelated, ie, the chronological order is unreasonable and the response not consistent with known adverse drug reactions, the patient's disease or other treatment can also cause such results, the disease state is improved or the response eliminated when other treatments are stopped, and the reactions are reproduced when other treatments are repeated.

\section{Treatment of adverse events}

The investigators used AE terminology and accurate medical terminology to record the following conditions of AE components on the adverse-reaction observation page in the case-report form: symptom description, date of occurrence, date of symptom discontinuation, degree, measures taken, evaluation of the relationship with the drug, and the final outcome. For all AEs, it was necessary to track and obtain sufficient information to determine their consequences. If an AE or its consequences persisted, follow-up was continued until the AE had disappeared or stabilized.

\section{Bioanalytical methodology Determination of Svt and its metabolite}

In this study, a validated liquid chromatography (LC)-mass spectrometry (MS)/MS method was developed to determine the human plasma concentration of Svt and its metabolite: Svt acid (SvtA). Svt and SvtA were separated by a Poroshell 120 EC-C ${ }_{18}$ column $(50 \times 4.6$ mm, $2.7 \mu \mathrm{m}$; Agilent Technologies, 
Table I Gradient condition of LC for Svt and SvtA

\begin{tabular}{l|l|l|l}
\hline $\begin{array}{l}\text { Time } \\
\text { (minutes) }\end{array}$ & $\begin{array}{l}\text { Flow rate } \\
(\mathbf{m L} / \mathbf{m i n})\end{array}$ & A (\%) & B (\%) \\
\hline Initial & 0.80 & 60 & 40 \\
2.2 & 0.80 & 10 & 90 \\
3.3 & 0.80 & 10 & 90 \\
3.4 & 0.80 & 60 & 40 \\
4.0 & 0.80 & 60 & 40 \\
\hline
\end{tabular}

Notes: ${ }^{2} 20 \mathrm{mmol}$ ammonium acetate; bacetonitrile.

Abbreviations: LC, liquid chromatography; Svt, simvastatin; SvtA, Svt acid.

Santa Clara, CA, USA) using gradient elution, and the mobile phase consisted of acetonitrile and aqueous phase (containing $20 \mathrm{mmol}$ ammonium acetate). Here, lovastatin (Lvs) and lovastatin acid were chosen as internal standard (IS). In order to improve the sensitivity and accuracy of detection, Svt and Lvs ionization was positive, while SvtA and Lvs acid were negative. Specific LC and MS conditions are shown in Tables 1-4.

\section{Plasma-sample processing of Svt and SvtA}

Plasma $(100 \mu \mathrm{L})$ was put into a $7 \mathrm{~mL}$ Eppendorf tube and $25 \mu \mathrm{L}$ IS solution added, with $150 \mu \mathrm{L}$ acetonitrile and $100 \mu \mathrm{L}$ in the mobile phase $(20 \mathrm{mmol}$ ammonium acetate solution). After vortexing for 5 minutes, $3 \mathrm{~mL}$ methyl tert-butyl ether was added and vortexed for 10 minutes continually, followed by centrifugation at 5,000 rpm for 10 minutes. The supernatant was put into a $10 \mathrm{~mL}$-tip Eppendorf tube and the solvent evaporated under nitrogen at $40^{\circ} \mathrm{C}$. The precipitate was reconstituted by $200 \mu \mathrm{L} 90 \%$ acetonitrile.

\section{Determination of $\mathrm{Bbr}$ and $\mathrm{FbtA}$}

Plasma concentrations of Bbr and Fbt acid (FbtA; metabolite of Fbt) were measured using a validated LC-MS/MS method. The analytical column was Poroshell $120 \mathrm{C}_{18}(50 \times 4.6 \mathrm{~mm}$, $2.7 \mu \mathrm{m}$; Agilent), and the mobile phase comprised acetonitrile and $0.1 \%$ formic acid. The flow rate was $0.6 \mathrm{~mL} / \mathrm{min}$. Here, tetrahydropalmatine was chosen as IS. Bbr, FbtA, and IS ionization was positive. Specific LC and MS conditions are shown in Tables 1-4.

Table 2 Transition reactions of Svt, SvtA, and IS (Lvs, LvsA)

\begin{tabular}{l|l|l|l|l}
\hline Molecule & Transitions & $\begin{array}{l}\text { Lon- } \\
\text { spray } \\
\text { voltage }\end{array}$ & $\begin{array}{l}\text { Declustering } \\
\text { potential }\end{array}$ & $\begin{array}{l}\text { Collision } \\
\text { energy } \\
(\mathbf{e V})\end{array}$ \\
\hline Svt & $436.4 \rightarrow 199.1$ & 5,500 & 60 & 8 \\
SvtA & $435.3 \rightarrow 318.9$ & $-4,500$ & -70 & -23 \\
Lvs & $405.2 \rightarrow 199.1$ & 5,500 & 77 & 10 \\
LvsA & $421.2 \rightarrow 318.9$ & $-4,500$ & -80 & -22 \\
\hline
\end{tabular}

Abbreviations: Svt, simvastatin; SvtA, Svt acid; IS, internal standard; Lvs, lovastatin LvsA, Lvs acid.
Table 3 Gradient condition of LC for Bbr and FbtA

\begin{tabular}{l|l|l|l}
\hline $\begin{array}{l}\text { Time } \\
\text { (minutes) }\end{array}$ & $\begin{array}{l}\text { Flow rate } \\
(\mathbf{m L} / \mathbf{m i n})\end{array}$ & $\mathbf{A}(\%)^{\mathrm{a}}$ & $\mathbf{B}$ (\%) $^{\mathrm{b}}$ \\
\hline Initial & 0.60 & 70 & 30 \\
0.8 & 0.60 & 70 & 30 \\
1.0 & 0.60 & 20 & 80 \\
2.5 & 0.60 & 20 & 80 \\
2.6 & 0.60 & 70 & 30 \\
3.0 & 0.60 & 70 & 30 \\
\hline
\end{tabular}

Notes: ${ }^{\mathrm{a}} 0.1 \%$ formic acid; bacetonitrile.

Abbreviations: LC, liquid chromatography; Bbr, berberine chloride; FbtA, fenofibrate acid.

\section{Plasma-sample processing of $\mathrm{Bbr}$ and $\mathrm{FbtA}$}

Plasma $(400 \mu \mathrm{L})$ was put into a $10 \mathrm{~mL}$ Eppendorf tube and $20 \mu \mathrm{L}$ IS solution added. After vortexing for 1 minute, $4 \mathrm{~mL}$ extraction solvent (dichloromethane:diethyl ether 3:2) was added and vortexed for 5 minutes continually, followed by centrifugation at 5,000 rpm for 10 minutes. The supernatant was put into a $10 \mathrm{~mL}$-tip Eppendorf tube and the solvent evaporated under nitrogen at $40^{\circ} \mathrm{C}$. The residue was reconstituted with $150 \mu \mathrm{L}$ methanol.

\section{Pharmacokinetic assessments and statistical analysis}

Noncompartmental pharmacokinetic parameters - half-life, $C_{\text {max }}$, time to $C_{\text {max }}\left(t_{\text {max }}\right), \mathrm{AUC}_{0-t}, \mathrm{AUC}_{0-\infty}$, mean residence time, apparent volume of distribution $\left(\mathrm{V}_{z} / \mathrm{F}\right)$, apparent clearance $\left(\mathrm{Cl}_{z} / \mathrm{F}\right)$, and $d f$-were calculated from plasma concentrationtime data. All blood-collection time points met the requirements of the trial plan. Pharmacokinetic parameters of Bbr, Svt, SvtA, and FbtA in healthy subjects were calculated with DAS 2.1 software supplied by the Pharmacological Society of China (Beijing, China). All data are expressed as mean \pm SD and differences considered to be significant when $P<0.05$.

\section{Results \\ Study subjects}

A total of 60 subjects were enrolled in this study. All of them signed an informed-consent form and satisfied the inclusion and exclusion criteria. No obvious clinical abnormalities

Table 4 Transition reactions of $\mathrm{Bbr}$ and IS

\begin{tabular}{l|l|l|l|l}
\hline Molecule & Transition & $\begin{array}{l}\text { Lon-spray } \\
\text { voltage }\end{array}$ & $\begin{array}{l}\text { Declustering } \\
\text { potential(s) }\end{array}$ & $\begin{array}{l}\text { Collision } \\
\text { energy } \\
(\mathbf{e V})\end{array}$ \\
\hline $\mathrm{Bbr}$ & $336.0 \rightarrow 319.8$ & 4,500 & 65 & 40 \\
$\mathrm{FbtA}$ & $319.0 \rightarrow 232.9$ & 4,500 & 30 & 20 \\
IS & $356 . I \rightarrow 192.1$ & 4,500 & 85 & 37 \\
\hline
\end{tabular}

Abbreviations: Bbr, berberine chloride; IS, internal standard; FbtA, fenofibrate acid. 
Table 5 Demographic characteristics of study participants $(n=60)$

\begin{tabular}{|l|l|}
\hline & Mean \pm SD \\
\hline Age (years) & $28.5 \pm 4.32$ \\
\hline Height $(\mathrm{cm})$ & $175.2 \pm 8.53$ \\
\hline Body weight $(\mathrm{kg})$ & $68.45 \pm 8.24$ \\
\hline Body-mass index $\left(\mathrm{kg} / \mathrm{m}^{2}\right)$ & $23.74 \pm 2.56$ \\
\hline
\end{tabular}

occurred before administration, and the investigators agreed that these subjects would participate in the trial. Throughout the trial, subjects were allowed to move freely only in permitted areas, but could not leave the ward casually and could not touch other things, including cigarettes, alcohol, coffee, and snacks. Subject-related demographic data are presented in Table 5 .

\section{LC-MS/MS validation}

Novel LC-MS/MS methods were developed and validated to determine Bbr, Svt, SvtA, and FbtA concentrations in healthy subjects' blood. We obtained clear biological samples, beautiful chromatographic peaks, and stable measurement by comprehensively optimizing biological sample extraction, LC, and MS. The matrix effect of Svt and SvtA was determined. Representative chromatography of blank blood samples, blank blood spiked with corresponding analytes, or IS demonstrated the selectivity of assays. For Bbr, calibration curves were obtained over a concentration range of $0.05-50 \mathrm{ng} / \mathrm{mL}$. Intra- and interday precision was $<9.3 \%$ and $10.6 \%$, respectively. Accuracy was within $10.4 \%$ and $10.3 \%$, respectively. Mean recovery of $\mathrm{Bbr}$ at three concentrations was $>89.6 \%$. For Svt and SvtA, calibration curves were obtained over a concentration range of $0.1-10.0 \mathrm{ng} / \mathrm{mL}$. Intraday precisions was $<10.5 \%$ and $11.6 \%$. Interday precision was $<10.9 \%$ and $11.4 \%$. Accuracy was within $8.4 \%$ and $9.3 \%$. Mean recovery at three concentrations was $>94.6 \%$ and $99.5 \%$, respectively. For FbtA, calibration curves were obtained at concentration of 50-20,000 ng/mL. Intra- and interday precisions were $<8.3 \%$ and $8.8 \%$, respectively. Accuracy was within $6.4 \%$ and $7.2 \%$. Mean recovery at three concentrations was $>100.4 \%$. All in all, linear, precision, accuracy, recovery, matrix effect, stability, and carryover met the requirements of biological sample-analysis methods after detailed methodological verification. As such, these methods were applicable for in vivo drug-interaction analysis in subjects.

\section{Pharmacokinetic analysis}

Pharmacokinetic drug-drug interactions (DDIs) between $\mathrm{Bbr}, \mathrm{Svt}$, and Fbt, and profiles and parameters of the groups are shown in Figures 2-4 and Tables 6 and 7.
In the single-dose group, the pharmacokinetic parameters of Bbr did not change significantly when used in combination with Svt and Fbt. Similarly, there was no obvious change in the in vivo behavior of Svt and Fbt due to the addition of Bbr. In the multiple-dose group, Svt and Fbt increase the $t_{\text {max }}$ of Bbr by about 2.5-fold and decrease the $\mathrm{V}_{z} / \mathrm{F}$ and $\mathrm{Cl}_{z} / \mathrm{F}$ about threefold, while Bbr showed less impact on the pharmacokinetic behavior of Svt and Fbt. Additionally, there were no significant sex differences in the in vivo pharmacokinetic behavior of Bbr, Svt, or Fbt.

\section{Safety assessment}

A single dose of Bbr $300 \mathrm{mg}$ was well tolerated when administered alone or in combination with Svt or Fbt in healthy volunteers. Similarly, the multidose group also achieved good tolerance. There were no deaths, though SAEs occurred throughout the trial. There were no significant changes in terms of hepatic or renal function, creatine kinase, serum creatinine, AST, or ALT. The most commonly reported adverse reactions were gastrointestinal disorders in the Bbr studies. All subjects successfully completed the trial, and no one dropped out of the test because of bad tolerance.

\section{Discussion}

One of the important factors for the development of a new medicine is a timely, accurate, and standardized assessment of its potential DDIs in accordance with DDI guidelines. DDI can occur throughout the process of drug absorption, distribution, metabolism, and excretion. Generally speaking, DDI based on drug metabolism is a research hot spot. At present, more and more reports show that DDIs are related to transporters, so they are also one of the factors that should be investigated in the development of new drugs. Additionally, DDI may also alter the correlation of pharmacokinetics with pharmacodynamics. Therefore, we should pay more attention to DDI to improve the safety of clinical medication.

According to technical guidelines for clinical pharmacokinetics of chemical drugs, in principle, both males and females should be combined. The average number of males and females should be half each, in order to understand the pharmacokinetic characteristics of drugs in the body better and also to observe whether the pharmacokinetics of the drug show sex differences. The age of the subjects should generally be $18-45$ years. In this study, we appropriately relaxed the age to 50 years to ensure the accuracy of the test results and the smoothness of enrollment. The ratio of males to females was $1: 1$, and enrolled subjects were all $<45$ years old. 


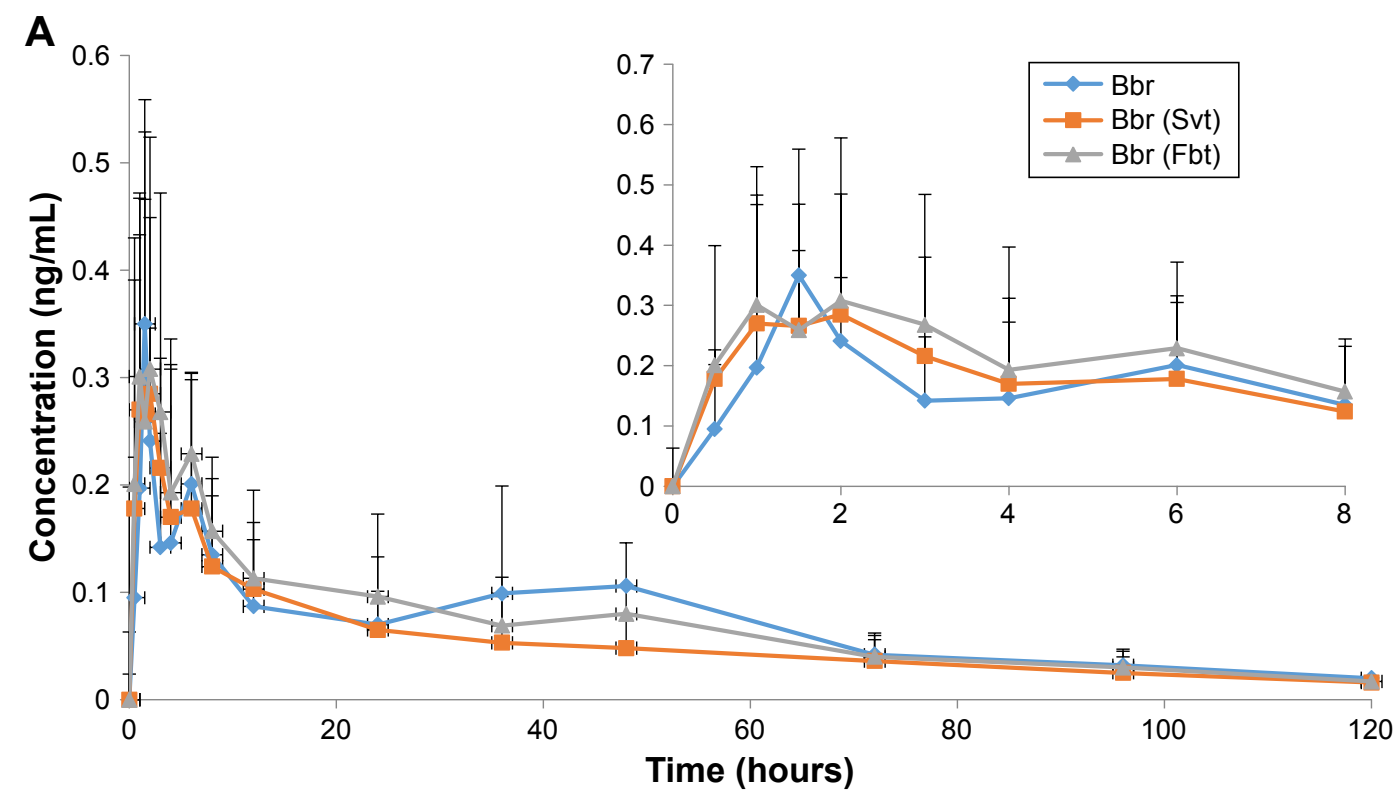

B

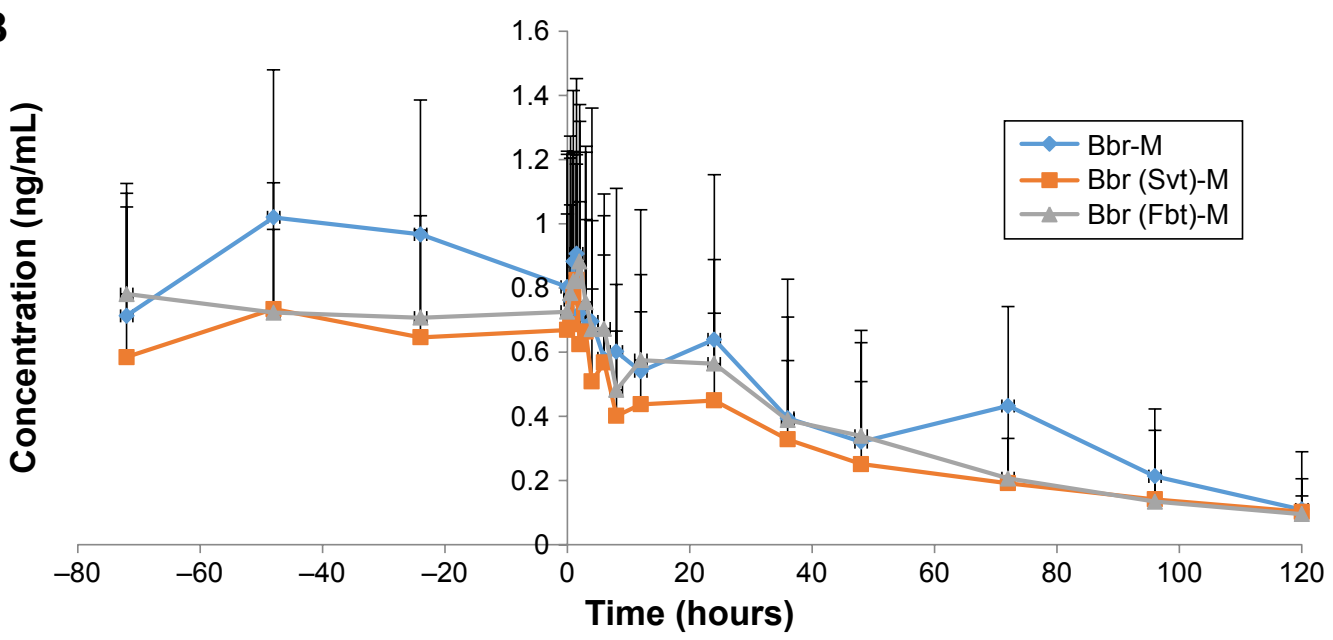

Figure 2 (A) Pharmacokinetic profiles of Bbr single-dose groups; (B) pharmacokinetic profiles of Bbr multiple-dose groups. Abbreviations: Bbr, berberine chloride; Svt, simvastatin; Bbr-M, berberine chloride multiple-dose group; Fbt, fenofibrate.

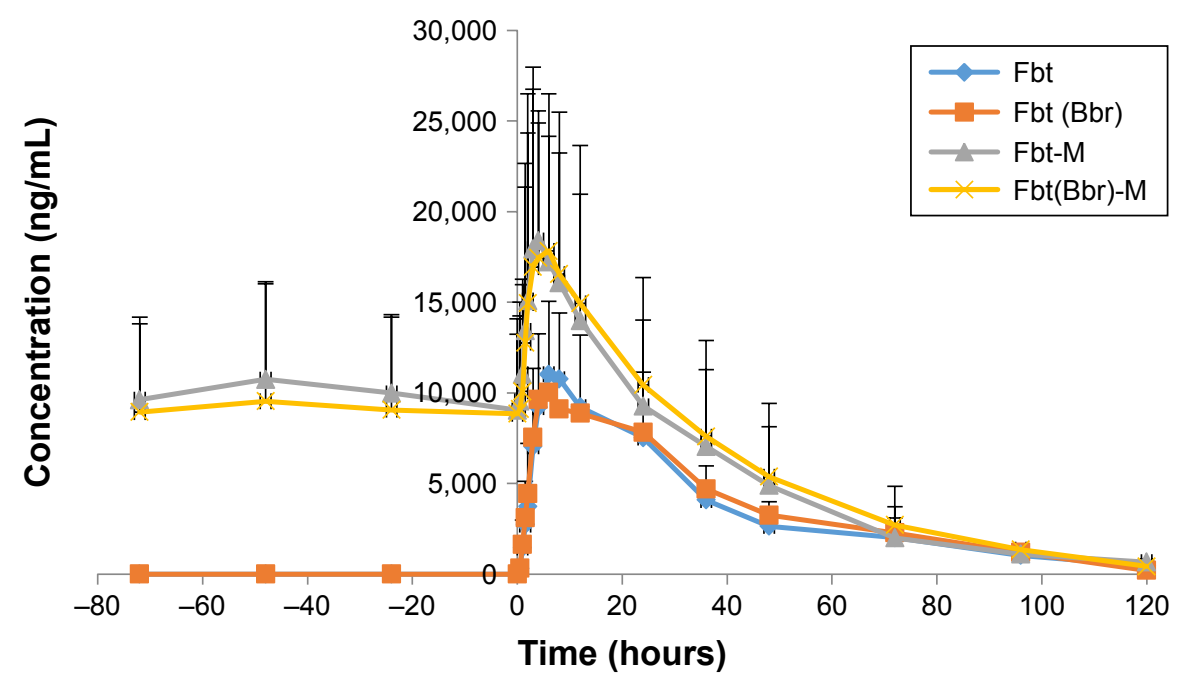

Figure 3 Pharmacokinetic profiles of Fbt single/multiple-dose groups.

Abbreviations: Svt, simvastatin; Bbr, berberine chloride; SvtA, Svt acid. 

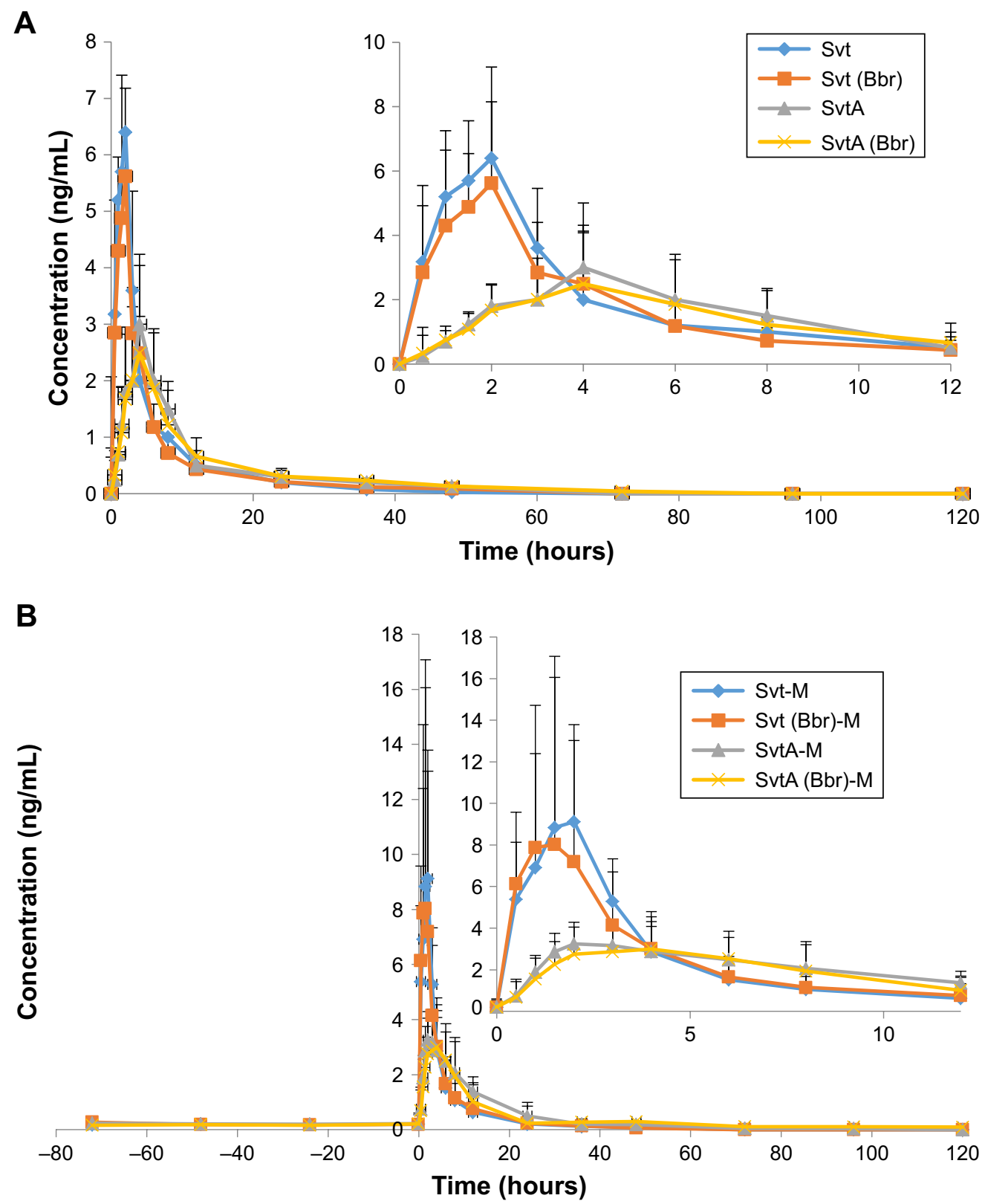

Figure 4 (A) Pharmacokinetic profiles of Svt and SvtA single-dose groups; (B) pharmacokinetic profiles of Svt and SvtA multiple-dose groups. Abbreviations: Svt, simvastatin; Bbr, berberine chloride; SvtA, Svt acid.

Table 6 Pharmacokinetic parameters of single-dose groups

\begin{tabular}{|c|c|c|c|c|c|c|}
\hline & \multicolumn{6}{|c|}{ Single-dose groups } \\
\hline & $t_{1 / 2}$ (hours) & $t_{\max }$ (hours) & $C_{\max }(\mu g / L)$ & $A^{\prime} \mathrm{C}_{0-\mathrm{t}}(\mu \mathrm{g} / \mathrm{L} \cdot \mathrm{h})$ & $\mathrm{AUC}_{0-\infty}(\mu \mathrm{g} / \mathrm{L} \cdot \mathrm{h})$ & $\mathrm{Cl}$ (L/h) \\
\hline $\mathrm{Bbr}$ & $30.60 \pm 23.68$ & $3.96 \pm 3.84$ & $0.33 \pm 0.24$ & $3.83 \pm 4.04$ & $6.28 \pm 6.06$ & $342,199 \pm 300,947$ \\
\hline Bbr (Svt) & $28.76 \pm 22.53$ & $3.13 \pm 3.16$ & $0.38 \pm 0.31$ & $3.83 \pm 4.04$ & $6.28 \pm 6.06$ & $298,543 \pm 233,655$ \\
\hline $\mathrm{Bbr}(\mathrm{Fbt})$ & $27.04 \pm 16.37$ & $3.63 \pm 1.85$ & $0.44 \pm 0.38$ & $4.48 \pm 4.73$ & $7.75 \pm 5.97$ & $286,833 \pm 304,009$ \\
\hline Svt & $4.65 \pm 2.98$ & $1.87 \pm 1.05$ & $6.93 \pm 4.76$ & $28.32 \pm 11.65$ & $30.18 \pm 12.83$ & $2,053 \pm 1,063$ \\
\hline SvtA & $4.26 \pm 1.87$ & $3.21 \pm 1.76$ & $3.22 \pm 0.67$ & $19.3 \pm 13.57$ & $21.02 \pm 15.33$ & $3,143 \pm 1,982$ \\
\hline Svt (Bbr) & $4.29 \pm 2.8$ & $1.58 \pm 0.95$ & $7.87 \pm 4.02$ & $25.55 \pm 10.32$ & $26.24 \pm 10.67$ & $|, 892 \pm|,|3|$ \\
\hline SvtA (Bbr) & $3.49 \pm 1.40$ & $2.59 \pm 1.65$ & $3.67 \pm 0.89$ & $20.3 \pm 16.73$ & $21.25 \pm 16.99$ & $3,074 \pm I, 88 \mid$ \\
\hline FbtA & $23.32 \pm 8.67$ & $6.88 \pm 4.76$ & $134,563 \pm 7,538$ & $305,376 \pm 142,365$ & $345,264 \pm 186,476$ & $0.88 \pm 0.43$ \\
\hline FbtA (Bbr) & $24.29 \pm 10.61$ & $5.75 \pm 3.22$ & $11,553 \pm 6,026$ & $286,590 \pm 131,209$ & $335,016 \pm 160,933$ & $0.74 \pm 0.37$ \\
\hline
\end{tabular}

Abbreviations: $\mathrm{AUC}$, area under the curve; Bbr, berberine chloride; $\mathrm{Cl}$, clearance; $\mathrm{C}_{\max }$, maximum concentration; Fbt, fenofibrate; FbtA, Fbt acid; Svt, simvastatin; SvtA, Svt acid; $t_{1 / 2}$, half-life; $t_{\text {max }}$, time to $C_{\text {max }}$. 


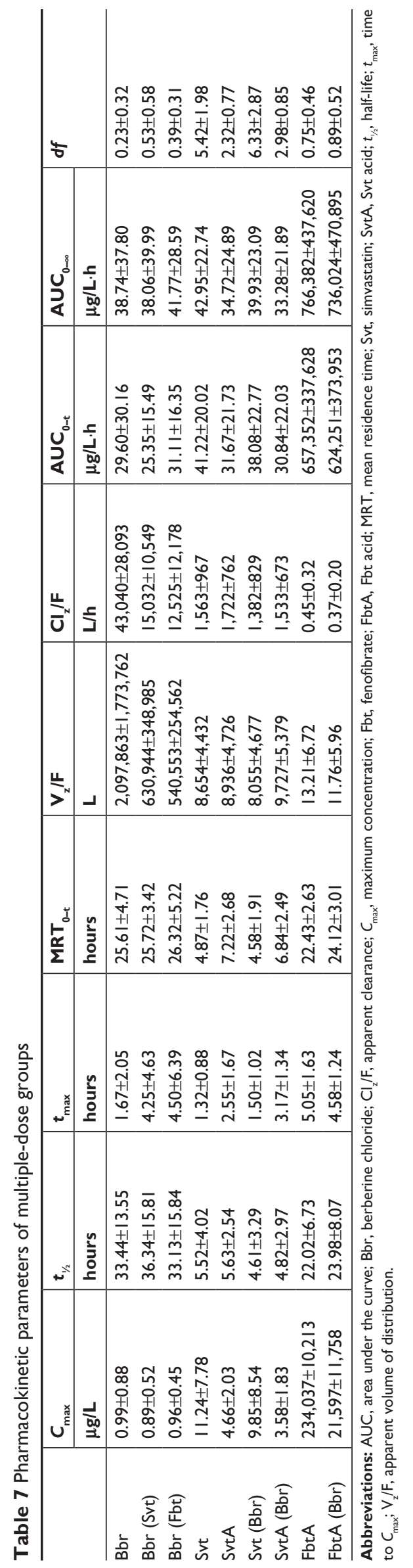

In the single-dose group, the pharmacokinetic parameters of Bbr did not change significantly when used in combination with Svt and Fbt. Similarly, there was no obvious change in the in vivo behavior of Svt and Fbt due to the addition of Bbr. This may have been due to: single administration not having a significant effect on metabolic enzyme activity; although Bbr, Svt, and Fbt are substrates for CYP3A4, competition among them is not obvious; or Bbr has more metabolic pathways and CYP1A2 has greater contribution compared with other metabolizing enzymes. The potential interaction between Bbr and Svt or Fbt was not significant when administered in a single dose.

In the multiple-dose group, Svt and Fbt increased the $t_{\max }$ of Bbr by about 2.5-fold and decrease $\mathrm{V}_{\mathrm{z}} / \mathrm{F}$ and $\mathrm{Cl}_{\mathrm{z}} / \mathrm{F}$ about threefold, while Bbr showed less impact on the pharmacokinetic behavior of Svt and Fbt. The reason may have been due to the following factors. As the time of administration increases, metabolic-based competition among Bbr, Svt, and Fbt is enhanced, because all three drugs are substrate for CYP3A4. Bbr has more phase I metabolic enzymes, the most important of which is CYP1A2. In addition, Fbt can downregulate the activity of OCT2-mediated organic anion transporters through independent PPAR $\alpha$ pathways, resulting in a decrease in the rate of Bbr transport into cells. According to the literature, although Svt and Fbt are substrates for the CYP3A4 enzyme, Svt can reduce the content and activity of CYP3A4 if in contact for a long time. At the same time, the metabolism of Svt would also be affected, due to its self-inhibition, while Fbt can also inhibit the metabolism of CYP3A4 on its substrate within a certain concentration range. In other words, the self-inhibition of Svt and Fbt enhances the effect of CYP3A4 on Bbr. In addition, the I phase metabolites of Bbr mainly include thalifendine and demethyleneberberine. For Bbr to thalifendine, CYP1A2, CYP2D6, and CYP3A4 are responsible for $78.38 \%, 18.97 \%$, and $2.65 \%$ of total transformation, respectively. For demethyleneberberine formation, CYP3A4 is responsible for $38.43 \%$, CYP1A2 for $31.18 \%$, and CYP2D6 for $30.39 \%$. It can be concluded that Bbr has multiple metabolic enzymes, and CYP3A4 is not a major metabolic one. All in all, the competitive effects of Svt and Fbt reduce the metabolism of Bbr by CYP3, leading to an increase in $t_{\max }$. Meanwhile, there were no obvious changes observed in bioavailability due to self-inhibition of Svt or Fbt or multichannel metabolism of Bbr. However, the half-life of Bbr did not change significantly, because it has more metabolic pathways and is excreted mainly by bile, with no obvious drug accumulation is found in the body. As for the decrease in $\mathrm{V}_{z} / \mathrm{F}$ and $\mathrm{Cl}_{\mathrm{z}} / \mathrm{F}$, this may have been related to Bbr. Bbr has obvious hepatic intestinal circulation effects 
and a long half-life. At the same time, the oral bioavailability of Bbr is low, only $5 \%$, so drug concentration in the body is at a lower level. The presence of a hepatic intestinal circulation effect caused the concentration of Bbr to fluctuate at a lower level, and the drug-time curve showed a significant multipeak phenomenon. This phenomenon brought great difficulties and deviations to the calculation of $\mathrm{V}_{\mathrm{z}} / \mathrm{F}$ and $\mathrm{Cl}_{\mathrm{z}} / \mathrm{F}$. In comparison, no significant difference in Bbr AUC was observed among groups, with the magnitude of changes in concentrations not being obvious.

Sex-dependent metabolism of drugs has been a hot point in the area of drug metabolism. It is helpful to learn about the sex-dependent metabolism of drugs in the rational use of drugs in clinics. As such, we also paid attention to whether there were sex differences in interactions among Bbr, Svt, and Fbt. The results showed no correlations between pharmacokinetic parameters and sex.

In this study, good drug tolerance and safety were obtained from DDI between Bbr and Svt or Fbt in healthy volunteers. Also, no deaths or SAEs occurred throughout the trial. All subjects successfully completed the trial, and no one dropped out because of bad tolerance. Long-term application of Svt and Fbt can lead to a decline in liver function and side effects such as rhabdomyolysis. These risks will be increased significantly as the dose is increased, especially when Svt and Fbt are combined, which may bring great inconvenience to patients. Bbr was tolerated well and did not undergo significant DDI when combined with Svt and Fbt in this study.

\section{Conclusion}

In this study, we investigated DDIs from the perspective of pharmacokinetics among Bbr, Fbt, and Svt and tolerability of combined administration in healthy Chinese subjects. It was found that potential interaction among Bbr, Svt, and Fbt was not significant when administered in a single dose. In the multiple-dose group, Svt and Fbt increased Bbr $t_{\max }$ about 2.5-fold and decreased $\mathrm{V}_{\mathrm{z}} / \mathrm{F}$ and $\mathrm{Cl}_{\mathrm{z}} / \mathrm{F}$ about threefold, but overall AUC did not change significantly, and no drug accumulation was observed. Bbr showed less impact on the pharmacokinetic behavior of Svt and Fbt. Additionally, concomitant administration of Bbr and Svt or Fbt was well tolerated by healthy subjects taking multiple doses over 7 days. These data are supportive of the coadministration of Bbr and Svt or Fbt. The current data indicate that DDIs among Bbr, Svt, and Fbt were mild and may not cause serious side effects, which would provide a new option for the treatment of hyperlipidemia.

\section{Acknowledgment}

Dr David B Jack is gratefully acknowledged for correcting the manuscript.

\section{Author contributions}

GL and LZ wrote the article; GL, MZ, and FQ designed the research; FQ and YS performed the research; and GL, MZ, and YS analyzed the data. All authors contributed to data analysis, drafting and revising the article, gave final approval of the version to be published, and agree to be accountable for all aspects of the work.

\section{Disclosure}

The authors alone are responsible for the content and writing of this article and report no conflicts of interest in this work.

\section{References}

1. Zhou X, Zhang W, Liu X, Zhang W, Li Y. Interrelationship between diabetes and periodontitis: role of hyperlipidemia. Arch Oral Biol. 2015;60(4):667-674.

2. Chung SD, Wang KH, Tsai MC, Lin HC, Chen CH. Hyperlipidemia is associated with chronic urticaria: a population-based study. PLoS One. 2016;11(3):e0150304-e0150309.

3. Maged Amin M, Rushdy M. Hyperlipidemia in association with proinflammatory cytokines among chronic spontaneous urticaria: casecontrol study. Eur Ann Allergy Clin Immunol. 2018;50(6):254-261.

4. Hylton Gravatt LA, Flurie RW, Lajthia E, Dixon DL. Clinical guidance for managing statin and antimicrobial drug-drug interactions. Curr Atheroscler Rep. 2017;19(11):46.

5. Abd El Aal HA, Ahmed LA, Hassan WA, Fawzy HM, Moawad H. Combination of carvacrol with simvastatin improves the lipid-lowering efficacy and alleviates simvastatin side effects. J Biochem Mol Toxicol. 2017;31(12):e21981.

6. Shinnakasu A, Yamamoto K, Kurano M, et al. The combination therapy of fenofibrate and ezetimibe improved lipid profile and vascular function compared with statins in patients with Type 2 diabetes. $J$ Atheroscler Thromb. 2017;24(7):735-748.

7. Tarantino N, Santoro F, de Gennaro L, et al. Fenofibrate/simvastatin fixed-dose combination in the treatment of mixed dyslipidemia: safety, efficacy, and place in therapy. Vasc Health Risk Manag. 2017;13: $29-41$.

8. Li YJ, Hu XB, Lu XL, et al. Nanoemulsion-based delivery system for enhanced oral bioavailability and caco- 2 cell monolayers permeability of berberine hydrochloride. Drug Deliv. 2017;24(1):1868-1873.

9. Fu K, Lv X, Li W, et al. Berberine hydrochloride attenuates lipopolysaccharide-induced endometritis in mice by suppressing activation of NF-KB signal pathway. Int Immunopharmacol. 2015;24(1):128-132.

10. Li D, Zheng J, Hu Y, et al. Amelioration of intestinal barrier dysfunction by berberine in the treatment of nonalcoholic fatty liver disease in rats. Pharmacogn Mag. 2017;13(52):677-682.

11. Li G, Yang F, Liu M, Su X, Zhao M, Zhao L. Development and application of a UPLC-MS/MS method for simultaneous determination of fenofibric acid and berberine in rat plasma: application to the drug-drug pharmacokinetic interaction study of fenofibrate combined with berberine after oral administration in rats. Biomed Chromatogr. 2016;30(7):1075-1082.

12. Ishii $\mathrm{Y}$, Ito $\mathrm{Y}$, Matsuki $\mathrm{S}$, et al. Clinical drug-drug interaction potential of BFE1224, prodrug of antifungal ravuconazole, using two types of cocktails in healthy subjects. Clin Transl Sci. 2018;11(5):477-486. 
13. Poór M, Boda G, Mohos V, et al. Pharmacokinetic interaction of diosmetin and silibinin with other drugs: Inhibition of CYP2C9-mediated biotransformation and displacement from serum albumin. Biomed Pharmacother. 2018;102:912-921.

14. Li Y, Liu L, Wang X, et al. In Vivo assessment of the effect of CYP1A2 inhibition and induction on pomalidomide pharmacokinetics in healthy subjects. J Clin Pharmacol. 2018;58(10):1295-1304.

15. Qiu W, Jiang XH, Liu CX, Ju Y, Jin JX. Effect of berberine on the pharmacokinetics of substrates of CYP3A and P-gp. Phytother Res. 2009; 23(11):1553-1558.

16. Wang Y, Zhang Y, Xiao J, Xu R, Wang Q, Wang X. Simultaneous determination of baicalin, baicalein, wogonoside, wogonin, scutellarin, berberine, coptisine, ginsenoside $\mathrm{Rb} 1$ and ginsenoside $\mathrm{Re}$ of Banxia xiexin decoction in rat plasma by LC-MS/MS and its application to a pharmacokinetic study. Biomed Chromatogr. In press 2018.

17. Follman KE, Dave RA, Morris ME. Effects of renal impairment on transporter-mediated renal reabsorption of drugs and renal drug-drug interactions: a simulation-based study. Biopharm Drug Dispos. 2018; 39(4):218-231.

18. Min JS, Bae SK. Prediction of drug-drug interaction potential using physiologically based pharmacokinetic modeling. Arch Pharm Res. 2017;40(12):1356-1379.
19. Lee SC, Arya V, Yang X, Volpe DA, Zhang L. Evaluation of transporters in drug development: Current status and contemporary issues. Adv Drug Deliv Rev. 2017;116:100-118.

20. Yao J, Kong W, Jiang J. Learning from berberine: treating chronic diseases through multiple targets. Sci China Life Sci. 2015;58(9): 854-859.

21. Motawi TK, Teleb ZA, El-Boghdady NA, Ibrahim SA. Effect of simvastatin and naringenin coadministration on rat liver DNA fragmentation and cytochrome P450 activity: an in vivo and in vitro study. J Physiol Biochem. 2014;70(1):225-237.

22. Tojcic J, Benoit-Biancamano MO, Court MH, Straka RJ, Caron P, Guillemette $C$. In vitro glucuronidation of fenofibric acid by human UDP-glucuronosyltransferases and liver microsomes. Drug Metab Dispos. 2009;37(11):2236-2243.

23. Asavapanumas N, Kittayaruksakul S, Meetam P, Muanprasat C, Chatsudthipong V, Soodvilai S. Fenofibrate down-regulates renal OCT2-mediated organic cation transport via PPAR $\alpha$-independent pathways. Drug Metab Pharmacokinet. 2012;27(5):513-519.

\section{Publish your work in this journal}

Drug Design, Development and Therapy is an international, peerreviewed open-access journal that spans the spectrum of drug design and development through to clinical applications. Clinical outcomes, patient safety, and programs for the development and effective, safe, and sustained use of medicines are the features of the journal, which has also been accepted for indexing on PubMed Central. The manuscript management system is completely online and includes a very quick and fair peer-review system, which is all easy to use. Visit http://www.dovepress.com/testimonials.php to read real quotes from published authors.

Submit your manuscript here: http://www.dovepress.com/drug-design-development-and-therapy-journal 